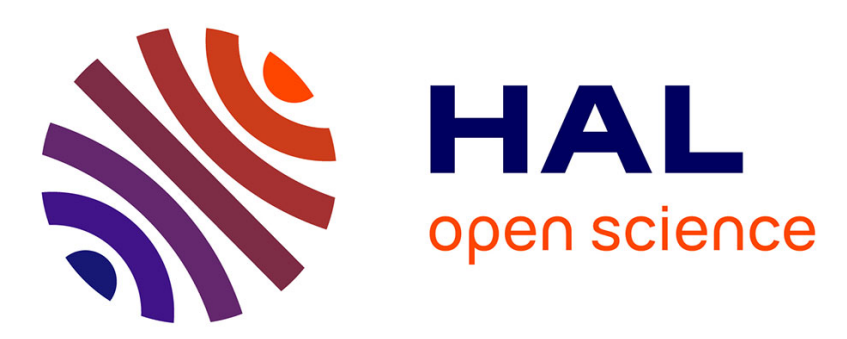

\title{
Void fraction in vertical gas-liquid slug flow: Influence of liquid slug content
}

Sébastien Guet, Sandrine Decarre, Veronique Henriot, Alain Liné

\section{To cite this version:}

Sébastien Guet, Sandrine Decarre, Veronique Henriot, Alain Liné. Void fraction in vertical gas-liquid slug flow: Influence of liquid slug content. Chemical Engineering Science, 2006, 61 (22), pp.7336-7350. 10.1016/j.ces.2006.08.029 . hal-01519969

\section{HAL Id: hal-01519969 \\ https://hal.science/hal-01519969}

Submitted on 9 May 2017

HAL is a multi-disciplinary open access archive for the deposit and dissemination of scientific research documents, whether they are published or not. The documents may come from teaching and research institutions in France or abroad, or from public or private research centers.
L'archive ouverte pluridisciplinaire HAL, est destinée au dépôt et à la diffusion de documents scientifiques de niveau recherche, publiés ou non, émanant des établissements d'enseignement et de recherche français ou étrangers, des laboratoires publics ou privés. 


\title{
Void fraction in vertical gas-liquid slug flow: influence of liquid slug content
}

\author{
S. Guet ${ }^{1}$, S. Decarre ${ }^{1, *}$, V. Henriot ${ }^{1}$ and A. Liné ${ }^{2}$ \\ ${ }^{1}$ Institut Français du Pétrole, Département Mécanique des Fluides, \\ 1 et 4 avenue de Bois-Préau, 92852 Rueil-Malmaison cedex, France. \\ ${ }^{2}$ Laboratoire d'Ingénierie des Procédés de l'Environnement, D.G.P.E., I.N.S.A., \\ 135, Avenue de Rangueil, $310^{\text {r7 }}$ Toulouse cedex 04, France. \\ *Corresponding author. E-mail address: sandrine.decarre@ifp.fr
}

\begin{abstract}
In this study we develop a model for computing the mean void fraction and the liquid slug void fraction in vertical upward gas-liquid intermittent flow. A new model for the rate of gas entrained from the Taylor bubble to the liquid slug is formulated. It uses the work done by the pressure force at the rear of the Taylor bubble. Then an iterative approach is employed for equating the gas entrainment flux and the gas flux obtained via conservation equations. Model predictions are compared with experimental data. The developed iterative method is found to provide reasonable quantitative predictions of the entrainment flux and of the void fraction at low and moderate liquid slug void fraction conditions. With an increased liquid slug void fraction, experimental data indicate that the flow in the liquid slug transits to churnheterogeneous bubbly flow thus gas entrainment flux tends to zero. Considering this effect in the iterative model significantly improved the predictions for large liquid slug void fraction conditions.
\end{abstract}

Key words: Gas-liquid, slug flow, slug aeration, gas entrainment, churn-bubbly, void fraction. 


\section{Introduction}

In the oil industry, two-phase gas-liquid flow in pipes often leads to intermittent (or slug) flow, for which a large gas-pocket is, intermittently, followed by a liquid slug. Above critical values of the superficial gas and liquid velocities, the liquid slug contains dispersed gas bubbles. In vertical gas-liquid intermittent flow the pressure gradient can be expressed by:

$$
\frac{\partial P}{\partial z}=\beta\left[\frac{\partial P}{\partial z}\right]_{P}+(1-\beta)\left[\frac{\partial P}{\partial z}\right]_{B},
$$

in which $\beta=\frac{L_{P}}{L_{P}+L_{B}}$ is the Taylor bubble length fraction $\left(L_{P}\right.$ is the length of the Taylor bubble region and $L_{B}$ is the length of the bubbly liquid slug region). In the Taylor bubble section the pressure is nearly constant thus $\left[\frac{\partial P}{\partial z}\right]_{P} \approx 0$. In the liquid slug section the pressure is essentially connected to the gravitational pressure (acceleration and friction contributions are small with respect to gravity). Provided the gas density is low with respect to the liquid density, the pressure gradient can be evaluated by

$$
\frac{\partial P}{\partial z} \approx(1-\beta) \epsilon_{L B} \rho_{L} g
$$

in which $\epsilon_{L B}$ is the liquid fraction in the slug, $\rho_{L}$ is the liquid density and $g$ is the acceleration of gravity.

Generally the liquid content in the Taylor bubble region is low in vertical gas-liquid flows (the liquid film thickness is small), therefore $\frac{\epsilon_{L P}}{\epsilon_{L B}}<<1$. Since the mean liquid fraction is given by $\epsilon_{L}=\beta \epsilon_{L P}+(1-\beta) \epsilon_{L B},(1-\beta) \epsilon_{L B} \approx \epsilon_{L}$ and the (gravitational) pressure gradient can be approximated by using the mean liquid fraction:

$$
\frac{\partial P}{\partial z} \approx \epsilon_{L} \rho_{L} g
$$

Therefore, using an empirical correlation for the mean void fraction in equation (3) can permit to successfully obtain a direct estimate of the pressure gradient in gas-liquid intermittent flow, without any required detailed parameter for the liquid slug. However equation (2) is in principle more accurate than (3) since no assumption on the liquid film thickness in the Taylor bubble region is needed. Furthermore in intermittent flow if the mean void fraction is to be modeled with a physically based approach, models for the flow in the Taylor bubble and in the liquid slug section should be used.

The aim of the present study is to develop a simplified physically based model for predicting the mean void fraction in the liquid slug $\epsilon_{G B}\left(=1-\epsilon_{L B}\right)$ and the mean void fraction $\epsilon_{G}\left(=1-\epsilon_{L}\right)$ in vertical co-current upward flow. Ultimately this model will be applied for mean liquid fraction and pressure gradient predictions. 
Slug aeration is essentially a function of the mixture velocity and of the tube diameter in horizontal flows and is correctly represented by the physically-based model of Andreussi and Bendiksen (1989) (Paglianti et al., 1991; Manolis et al., 1998; Orell, 2005). However inclination angle has a significant impact on slug aeration. For given flow conditions and fluid properties, liquid slug aeration increases significantly when increasing the inclination angle (Nydal, 1991; Nuland et al., 1997; Felizola and Shoham, 1995; Gomez et al., 2000; Zhang et al., 2003). As reported by Andreussi and Bendiksen (1989), their model for slug aeration is only valid for horizontal and slightly inclined flows, and does not take into account the significant increase of liquid slug void fraction with pipe inclination observed in the experiments.

To take into account the effect of pipe inclination on slug aeration, numerous semiempirical models have been suggested in the literature. Felizola and Shoham (1995) suggested various coefficients in a set of fitting laws to take into account the inclination effects. Tengesdal et al. (1999) used a model fitting approach to obtain the slug aeration as a function of inclination angle and of the phases superficial velocities. Gomez et al. (2000) proposed an exponential power law to describe the effects of inclination. Zhang et al. (2003) proposed a model for slug aeration based on the hypothesis that the entrainment and break-up of bubbles at the rear of the Taylor bubble was due to the additional pressure gradient associated with momentum exchange between the slug body and the film zone. This model provided promising results, however the slug length fraction was used as an input parameter. In this perspective, it is of interest to develop a physically-based model for slug aeration which does not use the slug length as an entry parameter.

Also physically-based models using the conservation of gas and liquid fluxes have been developed (Fernandes et al., 1983; Liné, 1983; Nydal, 1991; Brauner and Ullmann, 2004). In these models the aeration of the liquid slug is obtained by modeling the entrained gas flux at the rear of the Taylor bubble. A complete description of different regions of the flow and of the gas entrainment flux are needed in these models. The conservation of gas and liquid fluxes permits to close the model and to obtain predictions for the mean void fraction. In this study we want to develop and test such a physically based model for the prediction of liquid slug aeration and mean void fraction in vertically oriented upward intermittent flow. Improved models will be developed for describing the flow in the liquid slug and for the entrained gas flux, and experimental data will be used to check the validity of our models. The conceptual approach of Brauner and Ullmann (2004) based on an equilibrium of surface energy flux will be applied to model the gas entrainment flux at the rear of the Taylor bubble. Two mechanisms of gas entrainment will be considered: the turbulent 'jet' present at the rear of the Taylor bubble (Brauner and Ullmann, 2004), and the work done by the pressure jump at the rear of the Taylor bubble.

We consider that the flow can be separated in three parts (Brauner and Ullmann, 2004). These three parts are: the Taylor bubble region $(P)$, the Taylor bubble wake region $(W)$ and the developed liquid slug region $B \infty$ (figure 1). 
We assume that the flow is fully developed and at an equilibrium state, therefore the mean fluxes of gas and of liquid are equivalent at each boundaries:

$$
\psi_{G}=\psi_{G P}=\psi_{G W}=\psi_{G B \infty}
$$

in which the gas fluxes are defined as:

$$
\left\{\begin{array}{l}
\psi_{G}=\epsilon_{G}\left(V_{P}-V_{G}\right), \\
\psi_{G P}=\epsilon_{G P}\left(V_{P}-V_{G P}\right), \\
\psi_{G W}=\epsilon_{G W}\left(V_{P}-V_{G W}\right), \\
\psi_{G B \infty}=\epsilon_{G B \infty}\left(V_{P}-V_{G B \infty}\right) .
\end{array}\right.
$$

These fluxes are given in a frame of reference moving at the Taylor bubble nose velocity $V_{P} . \epsilon_{G}, \epsilon_{G P}, \epsilon_{G W}$ and $\epsilon_{G B \infty}$ are the mean void fraction in the whole section, in the Taylor bubble region, in the wake region and in the developed liquid slug region. $V_{G}, V_{G P}, V_{G W}$ and $V_{G B \infty}$ are the corresponding mean gas velocities.

In developed flow, also the liquid fluxes are equivalent, therefore

$$
\psi_{L}=\psi_{L P}=\psi_{L W}=\psi_{L B \infty}
$$

in which:

$$
\left\{\begin{array}{l}
\psi_{L}=\epsilon_{L}\left(V_{P}-V_{L}\right) \\
\psi_{L P}=\epsilon_{L P}\left(V_{P}-V_{L P}\right) \\
\psi_{L W}=\epsilon_{L W}\left(V_{P}-V_{L W}\right) \\
\psi_{L B \infty}=\epsilon_{L B \infty}\left(V_{P}-V_{L B \infty}\right) .
\end{array}\right.
$$

$\epsilon_{L}, \epsilon_{L P}, \epsilon_{L W}$ and $\epsilon_{L B \infty}$ are the mean liquid fraction in the whole section, in the Taylor bubble region, in the wake region and in the developed liquid slug region. $V_{L}, V_{L P}, V_{L W}$ and $V_{L B \infty}$ are the corresponding mean liquid velocities.

In the work of Brauner and Ullmann (2004), drift-flux relations with constant drift-flux parameters were used to model the gas and liquid velocity in the wake of the Taylor bubble and in the liquid slug. A model for the entrained gas flux at the rear of the Taylor bubble was suggested. The model of Brauner and Ullmann (2004) was shown to provide a reasonable description of slug aeration changes in inclined flows. Thus, such a model based on the conservation of gas and of liquid fluxes is expected to provide a robust approach for modeling the physics of slug flow hydrodynamics. However, as suggested by Brauner and Ullmann (2004), further research effort is required to establish the effect of the slug void fraction and tube inclination on appropriate values of the distribution parameter in aerated slug flow. The bubble entrainment phenomena at the rear of the Taylor bubble was attributed to the effects of the turbulent jet present at the rear of the Taylor bubble. Thus the turbulent kinetic energy of the jet present at the rear of 
the Taylor bubble was used to express the gas entrainment-flux (Brauner and Ullmann, 2004). It might however be expected that gas entrainment can be described by another mechanism: the pressure jump present at the rear of the Taylor bubble (Nydal, 1991), necessary to accelerate the liquid from the film flow around the Taylor bubble to the developed liquid slug.

In the present study we will develop improved closure models for the flow in the liquid slug and these models will be included in an iterative approach based on the conservation of phase fluxes. Experimental data will be analysed to infer appropriate models for the mean velocities in the Taylor bubble wake and in the liquid slug for vertical upward flow conditions, and physical considerations will be developed to obtain upper and lower limit values for the mean void fraction and for the gas entrainment flux. An alternative physical mechanism for gas entrainment will also be studied: the work done by the pressure force present at the rear of the Taylor bubble (Nydal, 1991). As will be shown, an advantage of this gas entrainment model compared to the jet model, is that the liquid velocity in the wake is not needed. Our developments will be included in an iterative approach, and the predictions will be compared with experimental data from Nydal (1991), Koeck (1980), Ferschneider (1982) and Fréchou (1986).

This manuscript is organised as follows: first, the experimental data used here will be presented. Upper and lower limit values for the void fraction and for the entrained gas flux will be derived and validated by experimental data. Also information about the mean gas velocity in the liquid slug and the entrained gas flux will be obtained from experimental data in section (2). This will enable us for conditioning our model and for developing appropriate gas velocity and flux models. In section (3) the basic principle of our model is described and the closure relations used in this study are explained. Possible gas entrainment mechanisms are discussed in section (4). Also criteria for the onset of gas entrainment will be discussed and formulated in this section. Then, we will make comparisons between experimental data and model predictions in section (5). Additional criteria for large void fraction conditions and large viscosity fluids will be suggested and compared with experiments in section (6).

\section{Experimental data bank and simplistic modeling}

\subsection{Experimental data}

The experimental data points used in the present study are the one of Nydal (1991), Koeck (1980), Ferschneider (1982) and Fréchou (1986). The data of Nydal (1991) and Koeck (1980) are in particular interesting for studying the effect of liquid input, surface tension properties and pipe diameter on liquid slug aeration. Since these data are concerned with air-water experiments, additional data are needed to study the effect of viscosity. The data of Ferschneider (1982) are used for this purpose. Also the ex- 
periments of Fréchou (1986) are interesting for viscosity and surface tension effects: although most of the experiments of Fréchou (1986) are conducted with a three phase flow of oil, water and gas, a few detailed experiments were reported for two phase flows of air and oil and of air and water. The same pipe was used in the two sets of experimental data.

Another interesting aspect of the data, is the way the experiments were achieved. In the experiments of Nydal (1991) and of Koeck (1980), the liquid input was kept constant and the gas input was increased progressively. Various liquid input conditions were studied using this approach (table 1). These particular conditions give us the opportunity of studying independently the effects of gas and liquid superficial velocities.

\subsection{Simplistic modeling}

\subsubsection{Void fraction upper and lower limits}

In vertical upward gas-liquid flows, the relative velocity between the gas and the liquid is positive (upward) in both the liquid slug part and in the gas-pocket, therefore the residence time of the gas-bubbles is smaller than the residence time of the liquid. In these conditions, the mean void fraction associated with no slip homogeneous flow conditions (noted with the subscript nos) constitutes an upper limit for the void fraction:

$$
\epsilon_{G, M a x}=\epsilon_{G, n o s}=\frac{U_{s g}}{U_{m}} .
$$

The gas flux in the frame of the moving Taylor bubble (at a velocity $V_{P}$ ), is given by $\psi_{G}=\epsilon_{G}\left(V_{P}-V_{G}\right)=\epsilon_{G} V_{P}-U_{s g}$. When there is no entrained bubble at the back of the Taylor bubble, the gas flux is zero (these conditions are noted with the subscript nof). At zero entrained gas-flux the mean void fraction reaches a minimum value, given by:

$$
\epsilon_{G, \min }=\epsilon_{G, n o f}=\frac{U_{s g}}{V_{P}} .
$$

It should however be noticed, that a zero gas entrainment rate does not neccesarly mean that the liquid slug is unaerated. Indeed, if the gas bubbles are convected at the same velocity as the Taylor bubble, $V_{P}=V_{G B}$ and $\psi_{G B}=\epsilon_{G B}\left(V_{P}-V_{G B}\right)=0$ without any required condition for $\epsilon_{G B}$.

In these relations, the Taylor bubble nose velocity is determined with the relation suggested by Nicklin et al. (1962):

$$
V_{P}=C_{0 P} U_{m}+0.35\left(\frac{\left(\rho_{L}-\rho_{G}\right) g D}{\rho_{L}}\right)^{\frac{1}{2}}
$$


in which $C_{0 P}$ depends on the flow conditions (Fabre and Liné, 1992):

$$
C_{0 P}=2.29\left[1-\frac{20}{E O}\left(1-e^{-0.0125 E o}\right)\right]
$$

for laminar flow, and

$$
C_{0 P}=\frac{\log R e_{m}+0.309}{\log R e_{m}-0.743}\left[1-\frac{2}{E o}\left(3-e^{-0.025 E o} \log R e_{m}\right)\right]
$$

for turbulent flow. Here $R e_{m}=\frac{U_{m} D}{\nu_{L}}$ and $E o=\frac{\rho_{L} g D^{2}}{\sigma}$. Typicaly, in turbulent flow with $R e_{m}>8000$ (ie. for air water flows at moderate and large mixture velocities), $C_{0 P}$ tends to 1.2 .

\subsubsection{Upper and lower limit estimates: validation}

Following the formulation of the upper and lower limits for the mean void fraction, the upper and lower bounds are given by equations 8 and 9 . As illustrated by figure 2 these expectations are validated by experimental data. In figure 3 the corresponding gravitational pressure gradient, calculated by using the lower and upper limits for the void fraction in equation 3, are presented. Although the pressure gradient can be approximated with this approach a more detailed model would be needed to gain accuracy in the pressure gradient estimates.

In figure 4 upper and lower limit estimates are compared with experimental data for the case of a viscous gasoil flowing through a pipe of diameter $D=7.37 \mathrm{~cm}$ (Ferschneider, 1982). Also in this case the upper and lower limit estimates are in accordance with experimental data. In this case the data points are generally well described by the upper limit, which corresponds to the no gas entrainment flux assumption. This is actually in agreement with observations reported for such large viscosity fluids, for which generally no aeration of the liquid slug is reported (Fréchou, 1986). In such case the liquid film flow in the Taylor bubble region is often laminar and the wake effects at the rear of the Taylor bubble are reduced (Campos et al., 1988; Pinheiro et al., 2000). These observations indicate that the gas entrainment flux tends to zero in such flow conditions.

\subsection{Mean gas velocity in the liquid slug}

In the liquid slug, the dispersed gas bubble velocity can be modeled by using the drift flux model (Zuber and Findlay, 1965):

$$
V_{G B}=C_{O B} U_{m}+V_{d r i f t} .
$$


In this relation the distribution parameter correctly describing the gas velocity is unknown for such conditions. Therefore, experimental data are used to infer appropriate drift-flux parameter models. Assuming no re-coalescence of bubbles, the gas-flux conservation equation leads to:

$$
\epsilon V_{P}-U_{s g}=\epsilon_{G B}\left(V_{P}-V_{G B}\right)
$$

We could not find any literature reporting direct measurements of the mean gas velocity in the liquid slug. However, using equation 14 the mean gas velocity in the liquid slug can be obtained :

$$
V_{G B}=V_{P}-\frac{\epsilon_{G} V_{P}-U_{s g}}{\epsilon_{G B}} .
$$

This relation permits to study the best-descriptive law for the mean gas velocity in the liquid slug, by using experimental data reporting simultaneous measurements of the mean void fraction and of the liquid slug void fraction. The experimental data of Koeck (1980) are used for this purpose.

As noted initially by Zuber and Findlay (1965) in their conclusion, "Plots in the velocity-flux plane which show abrupt change of slope and of intercept can be interpreted as indicating a change of flow regime". In heterogeneous bubbly flow conditions (or, similarly, in the "churn bubbly" regime), Zuber and Findlay (1965) suggested the use of the value $C_{0 B, \text { Churn }}=1.18$. Hibiki and Ishii (2003) proposed a similar value for the churn-bubbly flow, given by $C_{0 B, \text { Churn }}=1.2-\sqrt{\frac{\rho_{G}}{\rho_{L}}}$. This value is also comparable to the value of $C_{0 P}$ used in the Taylor bubble nose velocity (relation 10), therefore it can be expected that when operating in the churn bubbly regime, the mean gas velocity in the liquid slug will be comparable to the Taylor bubble nose velocity. For homogeneous bubbly flow, a value of order 1 was inferred by Zuber and Findlay (1965). For small bubbles and in the non-agitated bubbly flow, it was shown recently by Guet et al. (2003) and Guet and Ooms (2006) that at conditions where the radial distribution of void fraction is wall peaking the distribution parameter reaches a below unity value, of typically $C_{0 B}=0.95$.

In figure 5 the mean velocity in the slug bubble, calculated with equation 15 , is shown as a function of the mixture velocity for the experiments of Koeck (1980) at $U_{s l}=$ $0.7 \mathrm{~m} . \mathrm{s}^{-1}$. Also predictions using $C_{0 B}=0.95$ and $C_{0 B}=1.2$ are shown. As can be seen from figure 5 at increased mixture velocity the distribution parameter rises to a value of order 1.2. This result clearly indicates that the flow pattern in the liquid slug gradually evolves from a wall peaking, small bubble flow, to a core peaking churn-heterogeneous bubbly flow.

Another interesting aspect of this result, is that since the liquid slug gas velocity tends to the gas-pocket nose velocity, the gas flux given by $\psi_{G B}=\epsilon_{G B}\left(V_{P}-V_{G B}\right)$ tends to zero at increased mixture velocity. In figure 6 , the gas flux evaluated by using the mean 
void fraction $\left(\psi_{G}=\epsilon_{G} V_{P}-U_{s g}\right)$ is plotted as a function of the mixture velocity. Also the gas flux estimated by assuming an homogeneous wall peaking bubbly flow in the liquid slug $\left(C_{0 B}=0.95\right)$ is represented. In the bubbly flow regime the entrained gas flux increases with increased mixture velocity, and is well represented by using $C_{0 B} \approx 1$. However when the liquid slug is in the churn flow regime (ie. at $U_{m}>2 m . s^{-1}$ for the experiments of figure 6), the gas flux is nearly constant and even gradually decreases with an increased mixture velocity. This observation on the maximum gas entrainment flux is also supported by the results for the mean liquid fraction (figure 2). At large mixture velocity the mean liquid fraction tends to the zero gas flux assumption result $\left(\epsilon_{L} \approx 1-\epsilon_{G, \text { nof }}\right)$.

\section{Model formulation: vertical intermittent flow}

As explained in the introduction, an approach based on the conservation of phase fluxes is used. The approach suggested by Brauner and Ullmann (2004) is applied to separate the flow in three parts (figure 1): the Taylor bubble gas pocket $(P)$, the near wake zone in the liquid slug $(W)$, and the far wake zone $(B \infty)$. In this section we formulate models for the flow in these different regions.

\subsection{Taylor bubble region $(P)$}

We assume that the liquid film is free of gas and the liquid film flow is developed. The contribution of the nose region to the mean void fraction in the Taylor bubble section is neglected. The liquid film thickness is considered as small with respect to the pipe radius in vertical flow. Following these assumptions, the hydrodynamics of the liquid film can be described by a 2D falling film model. In addition the film flow is considered as fully developed and stationary. The force balance on a falling lump of fluid is then given by:

$$
\tau_{w}=\rho_{L} g \delta
$$

with $\delta$ the liquid film thickness and $\tau_{w}$ the shear stress at the wall. The wall shear stress is given by $\tau_{w}=\frac{1}{2} \rho_{L} f_{l} V_{L P}{ }^{2}$. For a laminar pipe flow $f_{l}=16 / R e_{f}$ and for a turbulent film flow we use Blasius relation, $f_{l}=0.079 R e_{f}^{-\frac{1}{4}}$. Here $R e_{f}=\frac{V_{L P} D_{h}}{\nu_{l}}$ is the film Reynolds number, based on the film hydraulic diameter $\left(D_{h}=4 \frac{A_{f}}{S_{f}}=4 \delta\right.$, with $S_{f}$ the wetted perimeter and $A_{f}$ the liquid cross sectional area). $\nu_{l}$ is the kinematic viscosity $\left(\nu_{l}=\frac{\mu_{l}}{\rho_{L}}\right)$. 
Neglecting the contribution of the Taylor bubble nose the mean void fraction in the Taylor bubble section is given by:

$$
\epsilon_{G P}=\left(\frac{D-2 \delta}{D}\right)^{2}
$$

Equation 16 and 17 are used to write a relation for the liquid film velocity $V_{L P}$, valid for a given liquid flux $\psi_{L}$. For a turbulent liquid film $\left(R e_{f}>1000\right)$,

$$
g D^{\frac{5}{4}}\left[1-\sqrt{1-\frac{\psi_{L}}{V_{P}-V_{L P}}}\right]^{\frac{5}{4}}=K_{\text {turb }} \nu^{\frac{1}{4}} V_{L P^{\frac{7}{4}}}
$$

with $K_{\text {turb }}=0.066$. For a laminar liquid film $\left(R e_{f}<1000\right)$,

$$
g D^{2}\left[1-\sqrt{1-\frac{\psi_{L}}{V_{P}-V_{L P}}}\right]^{2}=8 \nu_{l} V_{L P}
$$

\subsection{Developed liquid slug $(B \infty)$}

In the developed liquid slug, drift-flux relations are applied to compute the void fraction and phase velocities:

$$
V_{G B \infty}=C_{0 B \infty} U_{m}+U_{\infty} \epsilon_{L B \infty}{ }^{N}
$$

In the developed liquid slug $(B \infty)$, we assume the flow to be fully developed. It is first assumed that the liquid input conditions are such that turbulent break-up is determining the maximum bubble size in the developed liquid slug. Therefore, it might be assumed that at conditions where the liquid slug is aerated the corresponding bubble size is small and the radial distribution of void fraction in the liquid slug is wall peaking. As a result, we will first assume that the distribution parameter is constant and equal to $C_{0 B \infty}=0.95$.

The second term in the RHS of equation 20 is the weighted mean drift velocity and permits to take into account the effect of bubble drift velocity. In the present study this term is taken as $V_{d r i f t, B \infty}=U_{\infty} \epsilon_{L B \infty}{ }^{N}$ with $N=2.5$ as suggested by Zuber and Findlay (1965). The terminal velocity of a single bubble in an infinite medium, $U_{\infty}$, is given by (Harmathy, 1960): $U_{\infty}=1.53\left(\frac{\sigma g\left(\rho_{L}-\rho_{G}\right)}{\rho_{L}{ }^{2}}\right)^{\frac{1}{4}}$. 


\subsection{Wake region $(W)$}

In the Taylor bubble wake region, the entering liquid jet leads to a negative liquid velocity in the near wall area. On the contrary, in the central zone of the liquid wake liquid recirculation due to the large vortex will result in an increased liquid velocity. This assumption is supported by experiments: in the central part of the near wake region, the liquid velocity is oriented upward, while in the near wall region the liquid velocity is negative (van Hout et al., 2002). The typical length of this wake region is one to five pipe diameters (Pinheiro et al., 2000; van Hout et al., 2002; Sotiriadis and Thorpe, 2005).

Following this observation, we assume that in the wake region and at the pipe centerline, the liquid velocity is equal to the gas-bubble rise velocity. At $r=R-\delta$ ( $\delta$ is the thickness of the liquid film in the Taylor bubble region), it is assumed that the liquid velocity is zero. The minimum (negative) liquid velocity is taken as the (negative) liquid velocity of the liquid film. We also assume that the radial distribution of liquid velocity in the wake can be described by two parabolic functions. This assumption is consistent with available liquid velocity measurements in the wake of Taylor bubbles (van Hout et al., 1992, 2002; Nogueira, 2006), bubble column measurements (Mudde, 2005), as well as VOF-CFD calculations (Thorpe et al., 2001; Sotiriadis and Thorpe, 2005; Taha and Cui, 2006).

The radial distribution of liquid velocity is then given by:

$$
v_{l w}= \begin{cases}v_{l w, 1}=V_{P}\left[1-\left(\frac{r}{R-\delta}\right)^{2}\right] & \text { if } r<R-\delta \\ v_{l w, 2}=V_{L P}-4 \frac{V_{L P}}{\delta^{2}}\left[r-\left(R-\frac{\delta}{2}\right)\right]^{2} & \text { if } r>R-\delta .\end{cases}
$$

Based on this formulation, the mean liquid velocity is obtained:

using $V_{L W}=\frac{1}{A} \int_{A} v_{l w} d A$,

$$
V_{L W}=\frac{1}{2} \epsilon_{G P} V_{P}+V_{L P} \epsilon_{L P}-8 V_{L P} \frac{R^{2}}{\delta^{2}}\left[\frac{1}{4}-\frac{1}{4} \epsilon_{G P}{ }^{2}-\frac{2}{3} \tilde{r}_{m i n}\left(1-\epsilon_{G P} P^{\frac{3}{2}}\right)+\frac{1}{2} \epsilon_{L P} \tilde{r}_{\min }^{2}\right]
$$

in which $\tilde{r}_{\text {min }}=1-\frac{\delta}{2 R}$ is the (dimensionless) radial position of the minimum liquid velocity in the wake region.

This expression is found to provide reasonable estimates of the liquid velocity profile in the wake region. The shape of the functions (here parabolas) was not of primary importance for the mean liquid velocity determination: tests performed with flat profiles provided similar quantitative results for the mean liquid velocity in the wake. The most important aspect of this model is to consider the (negative) contribution of the liquid film velocity in the near wall region, on a layer of thickness $\delta$. As will be illustrated in 
section 5.1, this model is not equivalent to the use of a drift-flux model with a constant distribution parameter in the Taylor bubble wake zone.

\subsection{Closure formulation}

In the present approach, the model consists of 13 (independent) relations:

- 1 relation for the Taylor bubble nose rise velocity (equation 10),

- 3 relations for the gas flux conservation (equation 4),

- 3 relations for the liquid flux conservation (equation 6),

- for each of the 3 regions, hydrodynamics or drift flux models (equation 18 or 19, 20 and 22) (3 relations),

- 3 relations for linking the mean gas and the mean liquid fraction in each region $\left(\epsilon_{G i}+\epsilon_{L i}=1\right)$.

The unknowns of the system are: the Taylor bubble rise velocity, the mean void fraction in each of the three regions ( 3 unknowns), the mean liquid fractions ( 3 unknowns), the mean gas velocities (3 unknowns) and the mean liquid velocities (3 unknowns). Also the mean void fraction should be determined. Thus, there are 14 independent unknowns to determine and one additional relation is necessary for closing the model. This additional relation is introduced via a model for the rate of gas entrained at the rear of the Taylor bubble.

\section{Gas entrainment flux modeling}

\subsection{Principle}

In the present work it is assumed that the flux of surface energy is proportional to an energy supply, as suggested by Brauner and Ullmann (2004):

$$
E=\frac{6 \sigma}{d_{32}} \psi_{G e}
$$

in which $\psi_{G e}$ is the volumetric flux of entrained gas. At equilibrium and assuming no re-coalescence of bubbles into the Taylor bubble, the entrained gas flux $\psi_{G e}$ is equal to: $\psi_{G e}=\psi_{G}$.

We will further assume that the maximum stable bubble size $d_{\text {Max }}$, given by a critical Bond number $B o_{c}=\frac{\left(\rho_{L}-\rho_{G}\right) g d_{M a x}^{2}}{\sigma}=0.4$ (Brauner, 2001), is equal to the Sauter bubble diameter $d_{32}$. Similar relations are applied in the gas entrainment model of Brauner and Ullmann (2004) and of Zhang et al. (2003). 
Various energy sources are possible. In particular, it is possible that this energy supply $E$ is related to the turbulent 'jet' present at the rear of the Taylor bubble (Brauner and Ullmann, 2004), or to the work done by the pressure forces. These two possibilities are studied here. The associated flux of energy supply is formulated and the model is used with these two approaches to inspect the validity of the associated entrainment mechanisms.

\subsection{Entrainment flux due to turbulent jet (Brauner and Ullmann, 2004)}

At the back of the Taylor bubble, the liquid flow forms a jet, which can be characterised by a net (relative) jet velocity. In the model of Brauner and Ullmann (2004) the energy source is attributed to the turbulent jet:

$$
\frac{1}{2} \rho_{L} \sum \dot{u}^{2} \psi_{L}=\frac{6 \sigma}{d_{M a x}} \psi_{G e}
$$

Here $\dot{u}$ is the liquid velocity fluctuation, which is assumed to be related to the mean jet velocity: $\sum \dot{u}^{2}=0.03\left(V_{L W}-V_{L P}\right)^{2}$.

It is also expected that entrainment will only occur if the turbulent Weber number $W e_{t}=\frac{\rho_{L} d_{M a x} \sum \dot{u}^{2}}{3 \sigma}$ is above a critical value, taken as $W e_{c r i t}=\frac{2}{3}$ by Brauner and Ullmann (2004).

The model based on the turbulent jet assumption is then given by (Brauner and Ullmann, 2004):

$$
\psi_{G e}=\frac{1}{400 C_{J}} \frac{d_{M a x}}{D}\left(W e-W e_{c r i t}\right) \psi_{L}
$$

with a Weber number defined as $W e=\frac{\rho_{L} D\left(V_{L W}-V_{L P}\right)^{2}}{\sigma}$. More details about the turbulent jet entrainment model are given in the article of Brauner and Ullmann (2004).

\subsection{Entrainment due to pressure jump at the rear of the Taylor bubble}

We assume here that the pressure jump at the back of the Taylor bubble is responsible for gas entrainment. Thus, the gas entrainment flux results from a balance between the work done by pressure forces necessary to accelerate the liquid and the surface energy flux due to surface tension. 


\subsubsection{Pressure jump energy source}

Only the acceleration pressure drop at the rear of the Taylor bubble is considered. The pressure drop necessary to accelerate the liquid from the gas-pocket section to the (developed) liquid slug is given by (Nydal, 1991):

$$
\Delta P=\rho_{L}\left[\epsilon_{L P}\left(V_{L P}-V_{P}\right)^{2}-\epsilon_{L B \infty}\left(V_{L B \infty}-V_{P}\right)^{2}\right]
$$

which is re-written as

$$
\Delta P=\rho_{L} \psi_{L}\left(V_{L B \infty}-V_{L P}\right)
$$

\subsubsection{Critical conditions for entrainment}

Since we consider in this section, that the pressure jump at the rear of the gas bubble is responsible for gas entrainment, liquid slug aeration is governed by a competition between pressure forces and surface tension forces. Therefore, the onset of entrainment should be described by a critical number:

$$
\left[\frac{\Delta P}{\tau_{\sigma}}\right]_{c}=\left[\frac{\Delta P d_{M a x}}{\sigma}\right]_{c}=\frac{d_{M a x}}{D}[E u . W e]_{c},
$$

in which the Euler number $E u$ is given by $E u=\frac{\Delta P}{\rho_{L}\left(V_{L W}-V_{L P}\right)^{2}}$.

We consider that only the excess of energy present above a certain limit, ie. Eu.We > $[E u . W e]_{c}$, results in gas entrainment. The critical value $[E u . W e]_{c}$ should then verify the following condition: if there is no gas, $i e . U_{s g}$ is zero, the liquid slug is not aerated thus $[E u . W e]<[E u . W e]_{c}$ and $\psi_{G e}=0$. For each experimental data point a corresponding critical value $[E u . W e]_{c}$ is found by first calculating $E u$.We for $U_{s l}=U_{m}$, ie. for $U_{s g}=0$.

\subsubsection{Entrainment rate due to pressure jump}

It is assumed that a proportion $K_{\Delta P}$ of the work done by the pressure force is used for gas entrainment, and will then be available for surface energy transfer. Based on this assumption,

$$
K_{\Delta P} \rho_{L}\left(V_{L B \infty}-V_{L P}\right) \psi_{L}^{2}=C_{J} \frac{6 \sigma}{d_{M a x}} \psi_{G e} .
$$

The entrained gas flux is then given by:

$$
\psi_{G e}=K_{\Delta P} \rho_{L} \frac{d_{M a x}}{6 \sigma C_{J}}\left(V_{L B \infty}-V_{L P}\right) \psi_{L}^{2} .
$$


By including the critical conditions for entrainment (equation 28), the entrained gas flux is given by

$$
\psi_{G e}=\frac{K_{\Delta P}}{6 C_{J}} \frac{d_{M a x}}{D}\left(E u . W e-[E u . W e]_{c}\right) \psi_{L} .
$$

\section{4 comparison of the two gas entrainment approaches}

Comparing the turbulent jet and the pressure jump assumptions, the two formulations can be related by:

$$
\psi_{G e, \Delta P}=67 K_{\Delta P} E u \psi_{G e, J e t} .
$$

Thus the Euler number gives a direct proportionality between $\psi_{G e, \Delta P}$ and $\psi_{G e, J e t}$. A more detailed analysis of typical values taken by the Euler number showed that with our model and for the experiments considered in this study $E u \approx 0.2$, and $E u$ does not significantly vary. Thus, it can be expected that provided $K_{\Delta P}$ and $C_{J}$ are correctly evaluated equation 25 and 31 will provide sensibly similar results. However, advantages of the method based on the pressure jump assumption are: the simplicity of the critical conditions for gas entrainment, and the use of the liquid velocity in the developed liquid slug. Indeed, the mean liquid velocity in the wake region is not necessary in the pressure jump model (equation 31).

\subsection{Maximum gas entrainment}

In vertical upward intermittent flow, the Taylor bubble is having a net positive upward rise velocity. Therefore, the mean void fraction is always less than the mean void fraction given by an homogeneous no slip assumption: $\epsilon<\epsilon_{n o s}=\frac{U_{s g}}{U_{m}}$. Since in the flow conditions studied here $V_{G}$ and $\epsilon$ are by definition positive,

$$
\epsilon_{G, n o s} V_{G}>\epsilon_{G} V_{G}=U_{s g}>0
$$

Then the gas flux should verify

$$
\psi_{G}=\epsilon_{G}\left(V_{P}-V_{G}\right)<\epsilon_{G, \text { nos }}\left(V_{P}-V_{G}\right)<\epsilon_{G, \text { nos }} V_{P}-U_{s g} .
$$

Therefore, the gas flux has a maximum value:

$$
\psi_{G, M a x 1}=\epsilon_{G, n o s} V_{P}-U_{s g}
$$


In our gas entrainment model, these additional criterion are applied by using:

$$
\psi_{G, \text { entrainment }}=\min \left(\psi_{G, M a x 1}, \psi_{G e}\right) .
$$

In section 6 we will also consider a maximum packing value for the void fraction in the liquid slug, of $\epsilon_{G B, M a x}=0.55$. This leads to a maximum value of the entrainment flux:

$$
\psi_{G, \operatorname{Max} 2}=\epsilon_{G B, \operatorname{Max}}\left(V_{P}-V_{G B}\right)
$$

\subsection{Model implementation}

As listed in section 3.4, our approach consists in 14 unknown with 14 equations. It should be noticed, however, that when the pressure jump assumption is used to calculate the entrainment flux, the 4 flow properties of the wake region and its associated 4 equations are not necessary for computing the liquid slug void fraction and the mean void fraction. In this case the system of equation can therefore be reduced to 10 equations with 10 unknowns, and the wake flow parameters are obtained once the system of equations is solved.

Our model is implemented in an iterative loop. The objective of this iterative model is to reach the equality:

$$
\psi_{G}=\psi_{G, \text { entrainment }},
$$

in which the entrained gas-flux is calculated with the models explained above.

First the starting gas flux values are calculated by using a starting guess for the mean void fraction $\epsilon_{G}=\epsilon_{G \text {,start }}$. This starting value is taken as a mean value between the no gas-flux assumption $\left(\epsilon_{G, n o f}=\frac{U_{s g}}{V_{P}}\right)$, and the no-slip assumption $\left(\epsilon_{G, n o s}=\frac{U_{s g}}{U_{m}}\right)$ :

$$
\epsilon_{G, \text { start }}=\frac{1}{2}\left(\frac{U_{s g}}{V_{P}}+\frac{U_{s g}}{U_{m}}\right)
$$

Then, iterations are carried out on the gas-flux by using the model consisting of the relations discussed in section 3.4 and of the gas entrainment model. At each iteration a new gas flux is calculated, which is a weighted average value of the previously calculated value and of the entrained gas flux model result. Using this relaxation procedure permits to obtain a converged gas flux and prevents oscillations. Convergence criteria are applied to the model, based on a maximum relative deviation of the gas fluxes of $\frac{\Delta \psi_{G}}{\psi_{G}}$ and of the associated void fraction in the liquid slug $\frac{\Delta \epsilon_{G B}}{\epsilon_{G B}}$ of 0.001 . With these convergence criteria, typical calculations are taking 50 to 800 iterations. 


\section{Results}

In the present section we will discuss results obtained with the two gas entrainment models. The liquid slug content and mean liquid fraction are computed and compared with experimental data. The model used in this section is called the initial model.

\subsection{Equivalent distribution parameter in the wake region}

It is interesting to compare the results obtained with our model for the flow in the Taylor bubble wake with observations made in bubble column flows, where the liquid velocity is also reported to have negative values in the near-wall region. Hibiki and Ishii (2003) and Guet et al. (2004) report larger values of the distribution parameter in bubble column and low liquid input pipe flows compared to convected upward bubbly pipe flow. This is due to the presence of a returning flow in the near wall region. By using our method, we can calculate the equivalent distribution parameter in the wake by using the relation:

$$
C_{0 W}=\frac{V_{G W}-U_{\infty} \epsilon_{L W}}{U_{m}}
$$

Such a result for the distribution parameter in the wake zone is presented in figure 7 for an air-water flow in a pipe of diameter $D=10 \mathrm{~cm}$. Indeed, with our profiles for the liquid velocity in the wake, the equivalent distribution parameter reaches large values $\left(C_{0 W} \approx 1.4\right.$ at low input velocities). When the velocities are increased, the liquid down flow is eventually suppressed, resulting in a decreased distribution parameter.

\subsection{Void fraction}

\subsection{Void fraction in the liquid slug}

In figure 8, predictions are compared with the experimental data of Koeck (1980) and in figure 9 similar comparisons are reported for the experimental data of Nydal (1991). For the jet model equation 25 is applied with $C_{J}=1$ (Brauner and Ullmann, 2004). For the pressure jump model relation 31 is used and the coefficient $K_{\Delta P}$ was calibrated to the experimental data. All the data points were used for this purpose. The value $K_{\Delta P}=10^{-2}$ was found to correctly describe the experiments and will be used in all cases. As mentioned before the critical conditions for entrainment are computed by calculating Eu.We associated with $U_{m}=U_{s l}$. 
As illustrated by figures 8 and 9 , the comparisons between experimental data and iterative code predictions are reasonable. Generally the pressure jump hypothesis (equation 31 ) provides slightly better results than the jet model formulation (equation 25). These slight differences are attributed to the use of a calibrated constant $K_{\Delta P}$ and an improved model for the onset of entrainment. With adapted coefficients $C_{J}$ and $W e_{c}$ and improved models for the flow in the wake, it is expected that the two methods would provide more similar results, in view of equation 32.

\subsection{Mean liquid fraction}

Typical comparisons between developed liquid slug void fraction predictions and experiments were presented in figures 8 and 9 . The comparisons were reasonable by using $K_{\Delta P}=10^{-2}$. However the results are not as satisfying with respect to the mean liquid fraction. As illustrated by figure 10, at large mixture velocities the predictions are generally underestimating the mean liquid fraction by typically $-50 \%$ when the void fraction in the liquid slug is correctly predicted (the measurements of Koeck (1980) at $U_{s l}=0.7 \mathrm{~m} / \mathrm{s}$ are also presented in figure 10, as in figure 8).

These simultaneous comparisons of mean void fraction and of liquid slug void fraction suggest additional effects to influence the void fraction at large mixture velocities. Possible additional effects, which should be taken into account for proper comparisons with experimental data, are: the flow properties in the liquid slug, and the characteristics of the film flow. This will be the subject of the next section. 


\section{Additional considerations}

In this section additional considerations are addressed. Essentially, these considerations concern the properties of the flow in the liquid slug and the character of the flow in the annular liquid film:

- the flow regime in the liquid slug seems to be best described by a "churn agitated bubbly flow" model at large mixture velocity (section 2.3);

- with viscous liquids, the film flow properties might affect the gas entrainment value. This will be analysed with experimental data.

\subsection{Homogeneous-bubbly to churn-bubbly flow transition}

\subsubsection{Experimental data information}

As discussed previously in section 2.3, at some conditions the gas velocity in the liquid slug is best described by a "churn-agitated" model (figure 5) and the entrainment flux decreases with an increased gas input (figure 6). A change from homogeneous to heterogeneous flow regime is observed at increased void fraction, similarly to bubble column flow conditions (Léon-Becerril and Liné, 2001; Mudde, 2005). It is expected that the change from an homogeneous to an heterogeneous flow is, therefore, associated with an increased mean void fraction in the liquid slug. Since as discussed in section 2.3 these regimes are associated with different drift-flux parameter values, it is possible to detect this effect by estimating the equivalent distribution parameter for the flow in the liquid slug:

$$
C_{0 B}=\frac{V_{G B}-V_{d r i f t}}{U_{m}},
$$

in which the mean velocity in the liquid slug is obtained from the experimental data, using equation 15 with the measured values of $\epsilon_{G B}$ and of $\epsilon_{G}$.

In figure 11 the results for the equivalent distribution parameter in the liquid slug are presented. In this plot it was assumed that $V_{\text {drift }}=0.35 \sqrt{g D}$ (Zuber and Findlay, 1965). The errorbars were calculated by assuming $V_{\text {drift }}=0$. It is clear from this picture, that the flow evolves from an homogeneous, wall peaking radial distribution of void fraction with $C_{0 B} \approx 0.95$, to an heterogeneous churn flow regime with $C_{0 B} \approx 1.15$ at a typical critical value of the liquid slug void fraction of $\epsilon_{G B}=0.35$. This result also supports the consideration that the void fraction in the liquid slug is the most important parameter for determining the flow regime (experiments carried out at various liquid input conditions are reported on this plot). 


\subsubsection{Bubbly to churn-bubbly regime transition model}

A model for the distribution parameter changes with liquid slug void fraction is suggested here. We consider that the transition takes place at a critical value of the liquid slug void fraction given by $\epsilon_{G B, c r i t}=0.35$. To take into account the gradual changes from an homogeneous wall peaking bubbly flow to a churn agitated bubbly flow, we propose the use of the following relation:

$$
C_{0 B}=\frac{C_{0 B, w p}}{1+\left(\frac{\epsilon_{G B}}{\epsilon_{G B, \text { crit }}}\right)^{N}}+\frac{C_{0 B, \text { churn }}}{1+\left(\frac{\epsilon_{G B, \text { crit }}}{\epsilon_{G B}}\right)^{N}} .
$$

In this relation, $C_{0 B, w p}$ is the distribution parameter associated with wall peaking homogeneous bubbly flow and $C_{0 B, c h u r n}$ is the distribution parameter associated with churn-heterogeneous bubbly flow. As illustrated by figure 11, the changes of equivalent distribution parameter could be reasonably described by using this model. Here we used $N=7, C_{0 B, \text { churn }}=1.2$ and $C_{0 B, w p}=0.95$. Comparisons between associated predictions for the mean gas velocity and experiments of Koeck (1980) at $U_{s l}=0.7 \mathrm{~m} . \mathrm{s}^{-1}$ are presented in figure 12. The model provides a reasonable description of the changes of mean gas velocity in the liquid slug.

In our iterative model, this bubbly to churn flow transition consideration is implemented as follows: the drift-flux model value $C_{0 B}=0.95$ used for describing an homogeneous model is replaced by equation 42 . In this relation the value of the void fraction in the liquid slug $\epsilon_{G B, c h u r n}$ is taken from the previous iteration. Then, relation 13 is applied to compute the mean gas-velocity in the liquid slug. The associated gas-flux is calculated by using:

$$
\psi_{G B, \text { churn }}=\epsilon_{G B, \text { churn }}\left(V_{P}-V_{G B, \text { churn }}\right) .
$$

Using a larger value for $C_{0 B}$ results in an increased gas velocity in the liquid slug. With our model for the gas entrainment, this resulted in an increased void fraction in the liquid slug at large mixture velocity. The maximum packing condition (equation 37) was employed to limit the value of the liquid slug void fraction at large mixture velocity.

\subsubsection{Results with the bubbly to churn-bubbly flow transition model}

In figure 13 the gas entrainment calculated with and without this churn flow consideration, is compared with the experimental data of Koeck (1980) at $U_{s l}=0.7 m . s^{-1}$. Taking into account this additional condition clearly improves the gas entrainment estimates at large mixture velocity. It also permits to better predict the associated mean liquid fraction (figure 14). 
In figure 15, we report comparisons of model predictions with all the measurements of the mean liquid fraction in air water systems (Fréchou, 1986; Koeck, 1980). Both the initial model (without churn flow considerations) and the improved model predictions are displayed. It is clear from this result, that our model provides improved predictions of the mean liquid fraction, particularly at low liquid fraction conditions, where the void fraction in the liquid slug is large and the liquid slug operates in the churn-bubbly regime.

Only when the liquid viscosity was increased (eg. for the experiments of Ferschneider (1982) and of Fréchou (1986) with oil), our model is found to underpredict the liquid fraction. Additional considerations on the properties of the flow in the falling liquid film and in the bulk liquid slug are addressed in the next section.

\subsection{Influence of film flow properties: viscosity effects}

Depending on the character of the liquid film flow, it can be expected that gas entrainment will be observed or not. As was illustrated by figure 4, indeed for a number of experimental point the mean liquid fraction with the viscous fluid experiments of Ferschneider (1982) is well described by a no gas-flux assumption. To study this effect, experimental data associated with large viscosity liquids are analysed separately in this section. Model predictions with the initial model are compared with an improved model. In this improved model, we will assume that if either the liquid film or the bulk flow is laminar, gas entrainment is absent.

\subsubsection{Viscous effects: model implementation}

The conditions used for determining the laminar-turbulent transitions are the following:

- The film flow is turbulent for $R e_{f}>1000$ (Kockx, 2005).

- We consider that the bulk flow is turbulent provided $R e_{m}>1200$.

If the flow in the film or in the liquid slug bulk is laminar, we will consider that the entrained gas flux is zero.

\subsubsection{Viscous effects: results}

The experimental data of Fréchou (1986) with viscous oil are used to study the effects of liquid viscosity. These data are interesting for this purpose, since typical values associated with these experiments are $R e_{f i l m}=250-500$. Also, in these conditions no churn flow in the liquid slug is expected since $\epsilon_{G}<0.4$. It is thus possible to study separately the issue of liquid viscosity with these data. 
In figure 16 a comparison is made between the experimental data points and model predictions. The results obtained with the initial model are compared with results obtained by neglecting gas entrainment. Indeed, neglecting gas entrainment provides better predictions than the initial model. In this view, it is also interesting to notice that in numerous experiments with viscous fluids in moderate diameter pipes, the film Reynolds number takes values in the range $R e_{f}=200$ to 1500 , therefore the film flow is in the transition region. Detailed measurements in these conditions would be of support to study the changes of gas entrainment rate during film flow transition.

\section{Conclusions}

A new physically based model was developed for predicting the hydrodynamics of gas-liquid cocurrent vertical upward intermittent flow. This new model employs the framework of the Taylor bubble wake model suggested by Brauner and Ullmann (2004). It includes an explicit formulation for the liquid velocity in the Taylor bubble wake region, a drift-flux formulation for the developed liquid slug part, and an hydrodynamic film model for the Taylor bubble region. Additional considerations are addressed with respect to lower and upper limits of the mean void fraction. Experimental data were used to study the existing flow regime in the liquid slug. It is found that the flow transits from wall peaking homogeneous flow to churn agitated bubbly flow when the gas fraction in the liquid slug is increased.

The existence of gas entrainment for laminar conditions in the film zone is also considered, and our (iterative) model is used to test the validity of two assumptions for the mechanism of gas entrainment. These gas entrainment mechanism assumptions are: (1) gas entrainment is due to the inertial effects of the turbulent 'jet' present at the rear of the Taylor bubble (Brauner and Ullmann, 2004), or (2) gas entrainment is to be associated with the work done by the pressure force necessary to accelerate the liquid from the Taylor bubble film region to the developed liquid slug. From the results, it is found that these two approaches provide similar results, since they are proportional to similar quantities, ie. $\left[V_{L W}-V_{L P}\right]^{2}$ and $\left[V_{L B \infty}-V_{L P}\right]^{2}$. However the model based on the pressure jump hypothesis provided sensibly better results. An advantage of this new gas entrainment formulation is that the wake region model is not needed to obtain mean void fraction information. The developed formulation for gas entrainment also included a model for the onset of gas entrainment. It is based on the simple consideration that gas entrainment can only exist when the gas input is different from zero, and provided a good description of the experiments.

At low and moderate values of the void fraction, as suggested by Brauner and Ullmann (2004) the mean void fraction in the liquid slug is essentially the result of a competition between the above mentioned gas-entrainment mechanism and surface tension. Indeed, in these conditions the liquid down flow in the film is large enough to guarantee small bubbles and the flow in the liquid slug is best described by an homogeneous model 
based on the assumption of a wall peaking radial distribution of void fraction. When increasing the void fraction in the liquid slug, the bubble size increases and the flow is the liquid slug progressively transits to churn-agitated turbulent bubbly flow regime. The mean gas velocity is then best described by a churn flow model. At these large mixture velocity conditions, models for the mean gas velocity in churn flow are having a formulation similar to the rise velocity of the Taylor bubble, therefore $\psi_{G B}=\epsilon_{G B}\left(V_{P}-\right.$ $\left.V_{G B}\right)$ tends to zero. Also, in these conditions the void fraction in the liquid slug reaches values of the order of the maximum packing value. Taking into account these effects is found to be crucial for proper mean void fraction predictions at large liquid slug aeration. A new model for the changes in the drift-flux distribution parameter in the liquid slug was suggested and tested on the experimental data. It clearly improved the accuracy of the predictions. Also the characteristics of the flow in the liquid film and in the bulk of the liquid slug are found to affect the aeration rate of the liquid slug. When the film flow or the bulk of the liquid slug is laminar, no liquid slug aeration is observed. This was taken into account in our model.

Finally, we should like to address an additional consideration concerning the length of the wake region. When operating at large mixture velocity the wake length might become, in some conditions, non negligible in comparison with the liquid slug length (Campos et al., 1988; Pinheiro et al., 2000). Thus the mean void fraction in the wake might contribute to the void fraction in the liquid slug. In experiments, it can be expected that depending on the experimental techniques used, the wake contribution is included or not in the liquid slug content measurement results. More detailed measurements in the wake zone when operating in the churn agitated liquid slug regime would be of support to understand these effects.

\section{Acknowledgments}

S. Guet is grateful to F. Brucy, E. Heintzé, E. Duret, M. Gainville and Y. Peysson for their interest and their support during this study. 


\section{References}

Andreussi, P., Bendiksen, K. 1989. An investigation of void fraction in liquid slugs for horizontal and inclined gas-liquid pipe flows. International Journal of Multiphase Flow, 15, pp 937-946.

Brauner, N. 2001. The prediction of dispersed flows boundaries in liquid-liquid and gas-liquid systems. International Journal of Multiphase flow, 27, pp 885-910.

Brauner, N, Ullmann, A. 2004. Modelling of gas entrainment from Taylor bubbles. Part A: slug flow. International Journal of Multiphase flow, 30, pp 239-272.

Campos, J.B.L.M., Guedes de Carvalho, J.R.F. 1988. An experimental study of the wake of gas slugs rising in liquids. Journal of Fluid Mechanics, 196, pp 27-37.

Fabre J, Liné, A. 1992. Modeling of two-phase slug flow. Annual Review of Fluid Mechanics, 24, pp 2146.

Felizola, H., Shoham, O. 1995. A unified model for slug flow in upward inclined pipes. Journal of energy resources and technology, 117, pp 7-12.

Fernandes, R.C., Semiat, R., Dukler, A.E. 1983. Hydrodynamic model for gas-liquid slug flow in vertical tubes. AIChE Journal, 29, pp 981-989.

Ferschneider, G. 1982. Ecoulement gaz-liquide à poches et à bouchons dans les conduites de section circulaire. PhD thesis, Institut National Polytechnique de Toulouse, France.

Fréchou, D. 1986. Ecoulement triphasique huile-eau air. PhD thesis, Institut National Polytechnique de Toulouse, France.

Guet, S., Ooms, G., Oliemans, R.V.A., Mudde, R.F. 2003. Bubble injector effect on the gas-lift efficiency. AIChE Journal, 49, pp 2242-2252.

Guet, S., Ooms, G., Oliemans, R.V.A., Mudde, R.F. 2004. Bubble size effect on low liquid input drift flux parameters. Chemical Engineering Science, 59, pp 3315-3329.

Guet, S., Ooms, G. 2006. Fluid mechanical aspects of the gas-lift technique. Annual Review of Fluid Mechanics, 38, pp 225-249.

Gomez, L.E., Shoham, O., Taitel, Y. 2000. Prediction of slug liquid holdup: horizontal to upward vertical flow. International Journal of Multiphase Flow, 26, pp 517-521.

Harmathy, T.Z. 1960. Velocity of large drops and bubbles in media of infinite or restricted extent. AIChE Journal, 6, pp 281-288.

Hibiki, T., Ishii, M. 2002. Distribution parameter and drift velocity of drift-flux model in bubbly flow. Int. Journal of Heat and Mass Transfer, 45, pp 707-721.

Hibiki, T., Ishii, M. 2003. One dimensional drift-flux model for two-phase flow in a large diameter pipe. Int. Journal of Heat and Mass Transfer, 46, pp 1773-1790.

Hout, R. van, Shemer, L., Barnea, D. 1992. Spatial distribution of void fraction within a liquid slug and some other related slug flow parameters. International Journal of Multiphase Flow, 18, pp 831-845.

Hout, R. van, Gulitski, A., Barnea, D., Shemer, L. 2002. Experimental investigation of the velocity field induced by a Taylor bubble rising in stagnant water. International Journal of Multiphase Flow 28, pp 579-596.

Kockx, J.P. 2005. Gas entrainment by a liquid film falling around a stationary Taylor bubble in a vertical tube. International Journal of Multiphase Flow, 31, pp 1-24.

Koeck, C. 1980. Etude du frottement pariétal dans un écoulement diphasique vertical 
ascendant. PhD thesis, Université Paris 6, France.

Léon-Becerril, E. and Liné, A. 2001. Stability analysis of a bubble column. Chemical Engineering Science, 56, pp 6135-6141.

Liné, A. 1983. Écoulement intermittent de gaz et de liquide en conduite verticale. $\mathrm{PhD}$ thesis, Institut National Polytechnique de Toulouse, France.

Manolis, I.G., Mendes-Tatsis, M.A., Hewitt, G.F. 1998. Average liquid volumetric content of slug region, film region and slug unit in high pressure gas-liquid slug flow. Proc. of the International Conference on Multiphase Flow, Lyon, France.

Mudde, R.F. 2005. Gravity-driven bubbly flows. Annual Review of Fluid Mechanics, 37, pp 393-423.

Nicklin, D.J., Wilkes, J.O., Davidson, J.F. 1962. Two phase flow in vertical tubes. Trans. Inst. Chem. Engs., 40, pp 61-68.

Nogueira, S., Riethmuler, M.L., Campos, J.B.L.M., Pinto, A.M.F.R. 2006. Flow in the nose region and annular film around a Taylor bubble rising through vertical columns of stagnant and flowing Newtonian liquids. Chemical Engineering Science, 61, pp 845-857.

Nuland, S., Malvick, IM., Valle, A., Hedne, P. 1997. Gas fractions in slugs in dense gas two-phase flow from horizontal to 60 degrees of inclination. ASME Fluids Engineering Division Summer.

Nydal, O.J. 1991. An experimental investigation of slug flow. PhD thesis, University of Oslo, Department of Mathematics, Norway.

Orell, A. 2005. Experimental validation of a simple model for gas-liquid slug flow in horizontal pipes. Chemical Engineering Science, 60, pp 1371-1381.

Paglianti, A., Trotta, G., Andreussi, P., Nydal, O.J. 1991. The effect of fluid properties and geometry on void distribution in slug flow. BhR group meeting.

Pinheiro, M.N.C., Pinto, A.M.F.R., Campos, J.B.L.M. 2000. Gas hold-up in aerated slugging columns. Transaction of the Institute of Chemical Engineers, 78, part A, pp 1139-1146.

Sotiriadis, A.A., Thorpe, R.B. 2005. Liquid re-circulation in turbulent vertical pipe flow behind a cylindrical bluff body and a ventilated cavity attached to a sparger. Chemical Engineering Science, 60, pp 981994.

Taha, T. Cui, Z.F. 2006. CFD modelling of slug flow in vertical tubes. Chemical Engineering Science, 61, pp 676687.

Tengesdal, J.O., Sarica, C., Kaya, A.S. 1999. Flow-pattern transition and hydrodynamic modeling of churn flow. SPE Journal, 4(4), pp 342-348.

Thorpe, R.B., Evans, G.M., Zhang, K., Machnievsky, P.M. 2001. Liquid recirculation and bubble breakup beneath ventilated gas cavities in downward pipe flow. Chemical Engineering Science, 56, pp. 6399-6409.

Zhang, H.Q., Wang, Q., Sarica, C., Brill, J.P. 2003. A unified mechanistic model for slug liquid holdup and transition between slug and dispersed bubble flows. International Journal of Multiphase Flow, 29, pp 97-107.

Zuber, N., Findlay, JA. 1965. Average volumetric concentration in two-phase flow systems. J. Heat Transfer Trans. ASME Ser., 87, pp 453-468. 


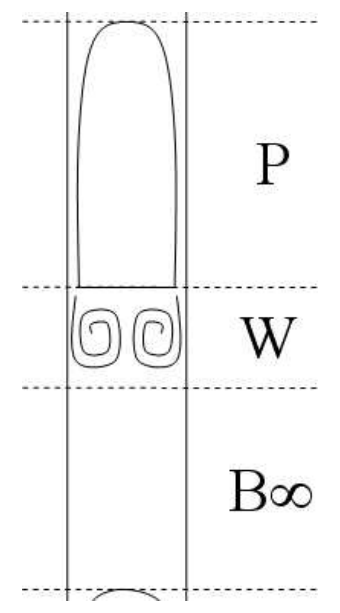

Fig. 1. Schematic principle of the model (Brauner and Ullmann, 2004). Three flow regions are considered: the Taylor bubble gas pocket $(P)$, the near wake zone in the liquid slug $(W)$ and the developed liquid slug region $(B \infty)$.

Table 1

Experimental data used in the present study.

\begin{tabular}{|c|c|c|c|c|c|c|}
\hline Author & $\sigma(\mathrm{mN} / \mathrm{m})$ & $\nu_{L}(\mathrm{mPa} . \mathrm{s})$ & $\rho_{L}\left(\mathrm{Kg} / \mathrm{m}^{3}\right)$ & $D_{P}(\mathrm{~cm})$ & $N_{\text {points }}$ & Specific conditions \\
\hline $\begin{array}{c}\text { Ferschneider (1982) } \\
\text { Air-oil }\end{array}$ & 34 & 10 to 35 & 887 & 7.37 & 24 & $P=10$ Bars \\
\hline Fréchou (1986) & & & & & & \\
Air-water & 46 & 1 & 1000 & 5.36 & 7 & $U_{s l}<1 \mathrm{m.s} \mathrm{s}^{-1}$ \\
Air-oil & 29 & 30 & 800 & 5.36 & 7 & $U_{s l}<1 \mathrm{m.s} \mathrm{s}^{-1}$ \\
\hline Koeck (1980) & & & & & & \\
Air-water & 57 & 1 & 1016 & 4.4 & 25 & $U_{s l}=0.4 ; 0.7 ; 1.1 ; 1.6 m . \mathrm{s}^{-1}$ \\
\hline Nydal (1991) & & 1 & & & & \\
Air-water & 72 & 1 & 1000 & 3.1 & 9 & $U_{s l}=1.2 m . \mathrm{s}^{-1}$ \\
\hline
\end{tabular}

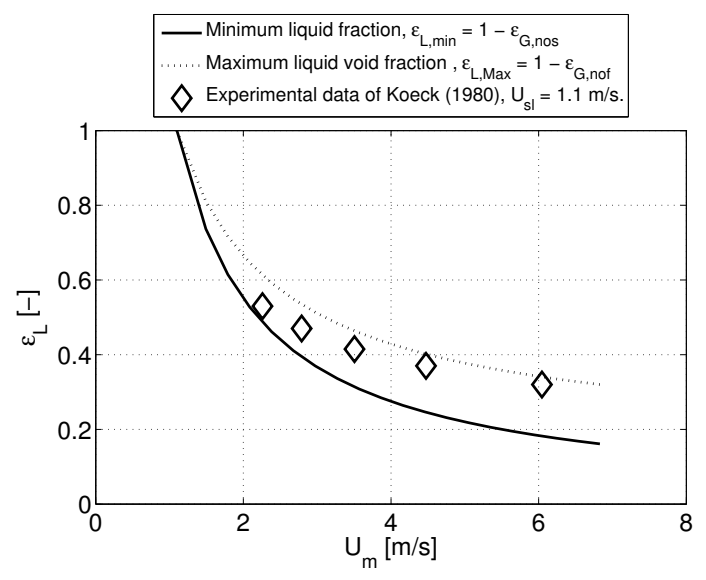

Fig. 2. Upper and lower limits for the liquid fraction and experimental data of Koeck (1980). In these experiments, $U_{s l}=1.1 \mathrm{~m} / \mathrm{s}$. 


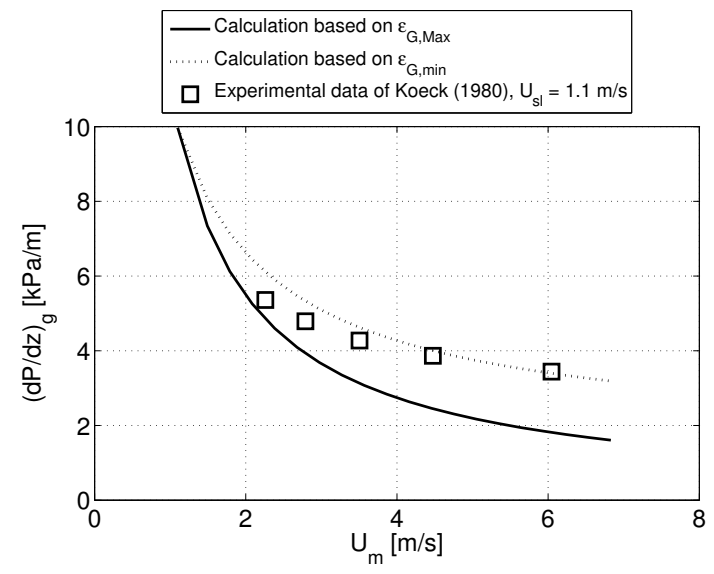

Fig. 3. Upper and lower limits for the gravitational pressure gradient by combining equation 3 with the upper and lower limit estimates for the void fraction. Also the experimental data of Koeck (1980) for $U_{s l}=1.1 \mathrm{~m} / \mathrm{s}$ are displayed, as in figure 2 .

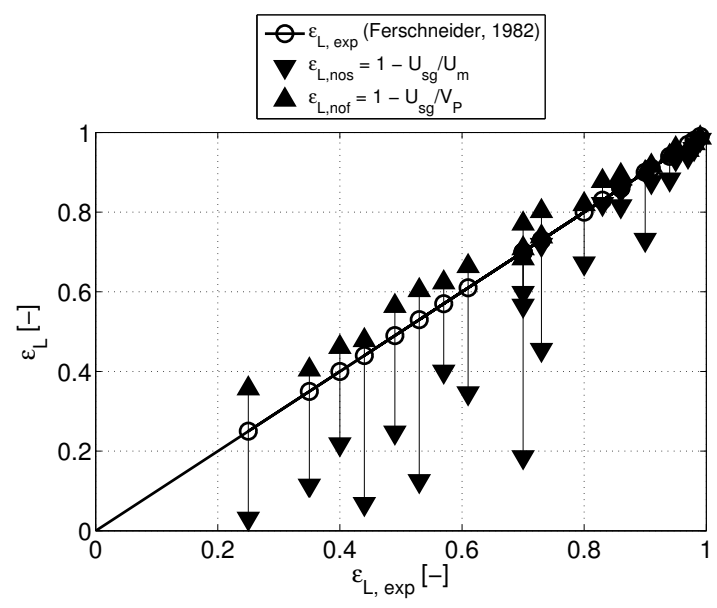

Fig. 4. Upper and lower limits for the liquid fraction for the experiments of Ferschneider (1982) with gasoil. Here the liquid viscosity is $\nu_{L}=10$ to $35 \mathrm{mPa} . \mathrm{s}$. 


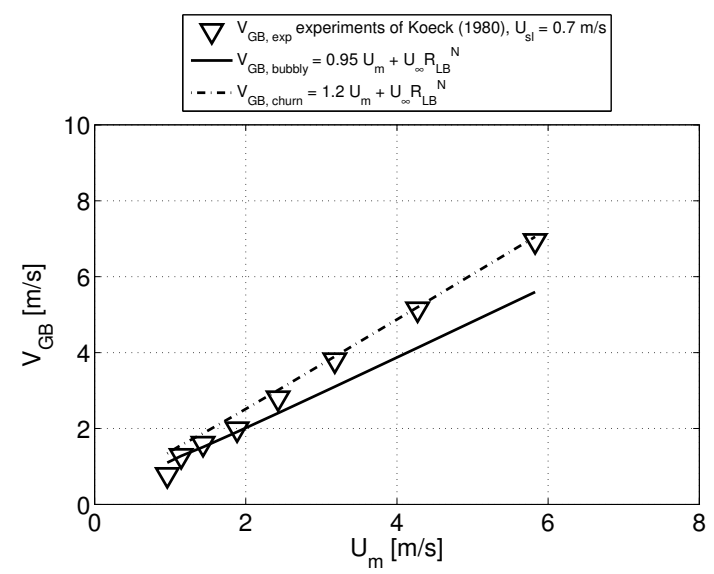

Fig. 5. Evaluated gas bubble mean velocity in the liquid slug, by using the experimental data of Koeck (1980) at $U_{s l}=0.7 m . s^{-1}$. The results are compared with the wall-peaking homogeneous bubbly flow assumption $\left(C_{0 B}=0.95\right)$ and a churn-bubbly heterogeneous flow model $\left(C_{0 B}=1.2\right)$.

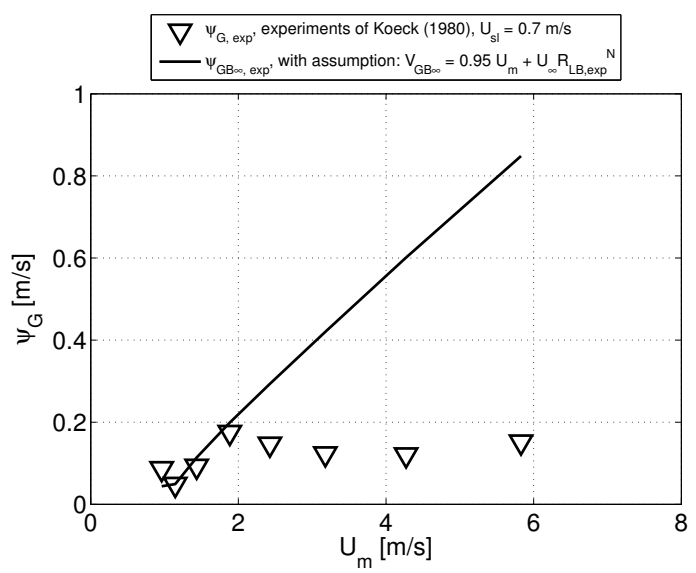

Fig. 6. Evaluated gas flux, by using the experimental data of Koeck (1980) at $U_{s l}=0.7 m . s^{-1}$. The correct gas flux $\psi_{G, \exp }=\epsilon_{G} V_{P}-U_{s g}$ (triangles) is compared with an estimate obtained via measurements of $\epsilon_{G B}$ and a wall-peaking drift-flux model assumption for $V_{G B}$ : $\psi_{G B, e x p}=\epsilon_{G B}\left(V_{P}-V_{G B}\right)$, with $C_{0 B}=0.95$ (line). 


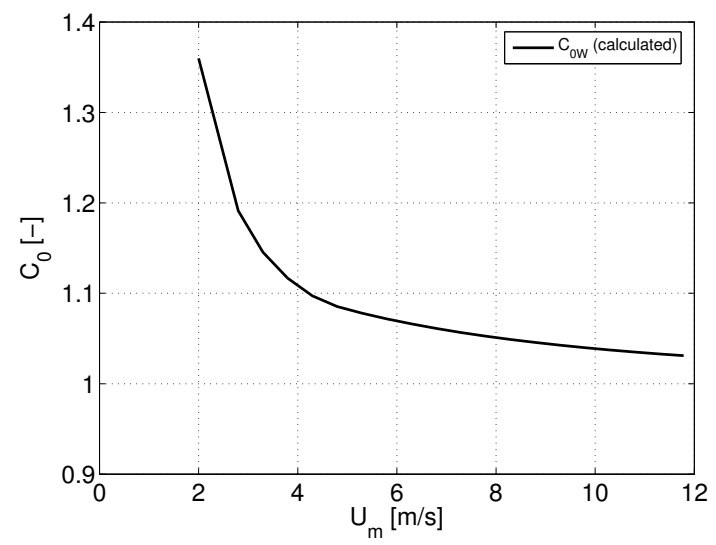

Fig. 7. Equivalent distribution parameter in the wake region, as a function of the mixture velocity. Here the conditions are: $D=10 \mathrm{~cm}, U_{s l}=2 \mathrm{~m} / \mathrm{s}$ with an air-water flow. The jet model of Brauner and Ullmann (2004) is applied for the gas entrainment rate.

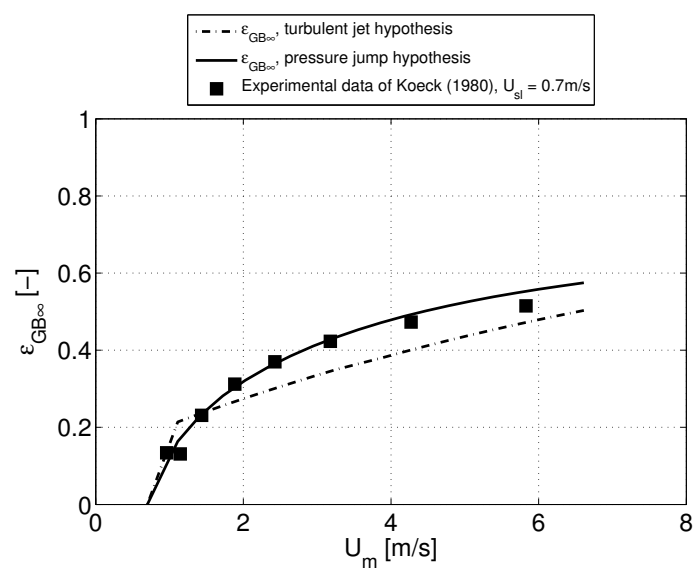

Fig. 8. Void fraction in the liquid slug as a function of the mixture velocity. Comparison with the experimental data of Koeck (1980) for $U_{s l}=0.7 \mathrm{~m} / \mathrm{s}$. 


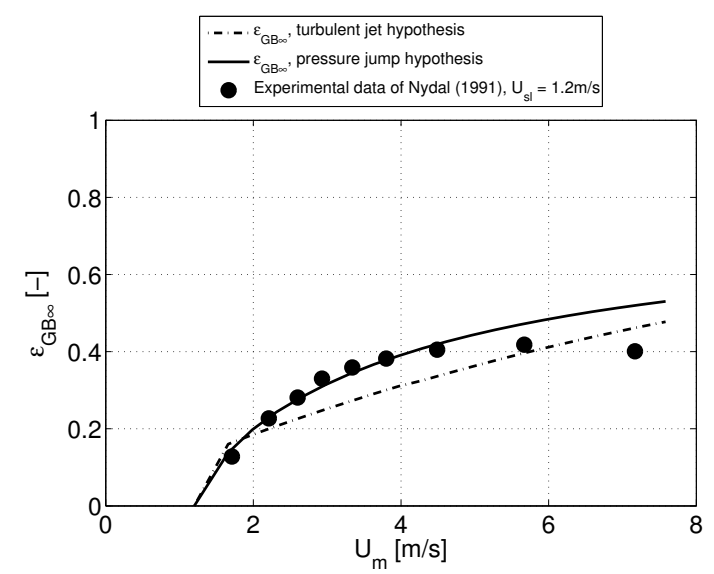

Fig. 9. Void fraction in the liquid slug as a function of the mixture velocity. Comparison with the experimental data of Nydal (1991) for $U_{s l}=1.2 \mathrm{~m} / \mathrm{s}$.

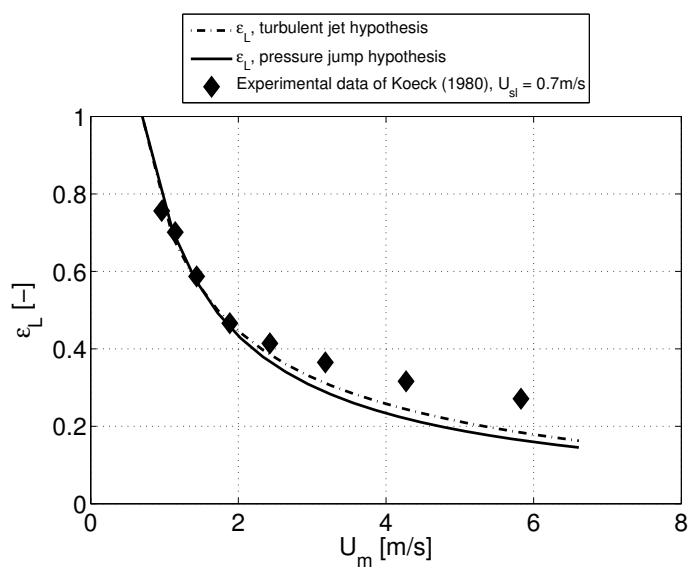

Fig. 10. Mean liquid fraction as a function of the mixture velocity. Comparison with the experimental data of Koeck (1980) for $U_{s l}=0.7 \mathrm{~m} / \mathrm{s}$ (same conditions as in figure 8). The liquid slug void fraction was correctly predicted, however the mean liquid fraction is underestimated at large mixture velocity conditions. 


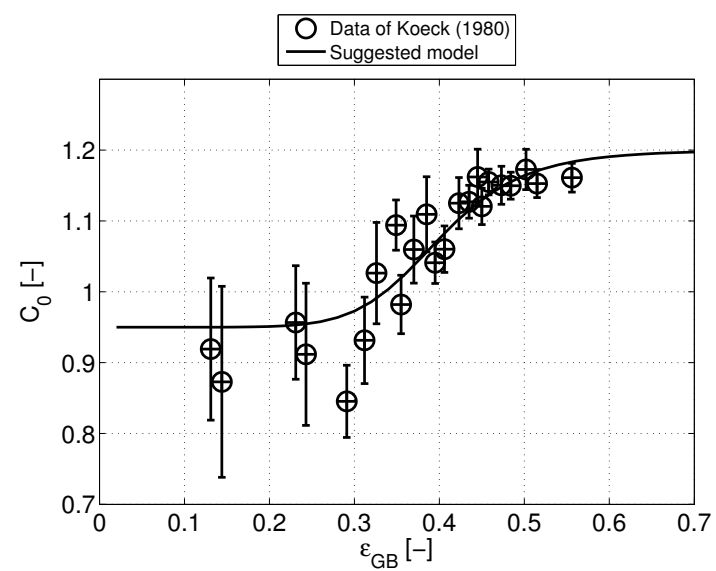

Fig. 11. Estimates of the equivalent distribution parameter in the liquid slug, as a function of the (measured) liquid slug mean void fraction. All the experimental data of Koeck (1980) are represented (ie. experiments for $U_{s l}=0.4 ; 0.7 ; 1.1$ and $1.6 \mathrm{~m} . \mathrm{s}^{-1}$ ). Also our suggested model is displayed (equation 42).

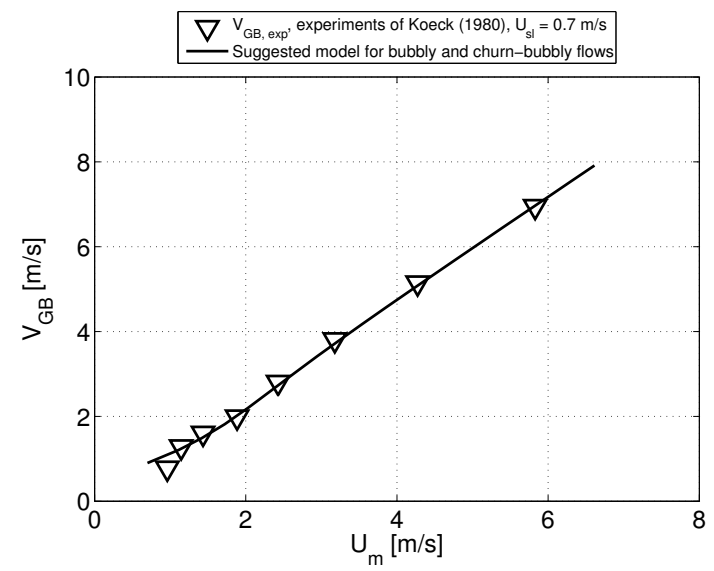

Fig. 12. Comparison between the mean gas velocity predicted by using our suggested model (relation 42) and the experimental data of Koeck (1980) at $U_{s l}=0.7 m . s^{-1}$. 


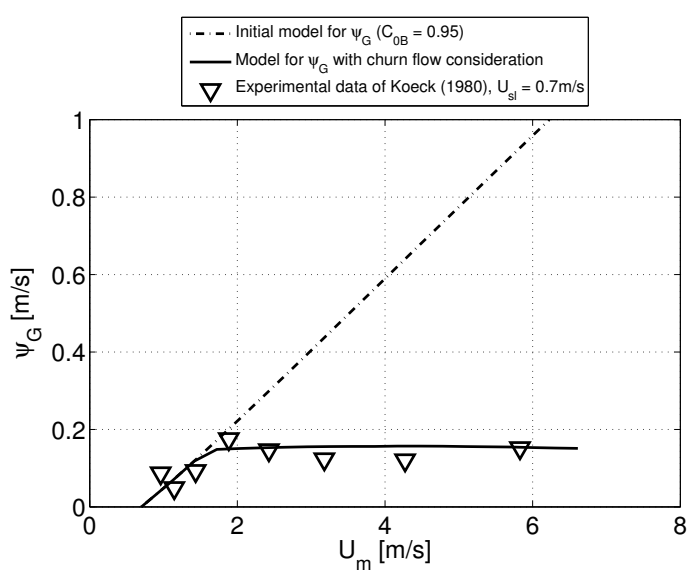

Fig. 13. Entrained gas flux obtained with the experimental data of Koeck (1980) at $U_{s l}=0.7 \mathrm{~m} \cdot \mathrm{s}^{-1}$ (triangles). Comparison with the gas entrained flux estimated with the initial homogeneous model and with the churn turbulent bubbly flow model suggested above (equation 43).

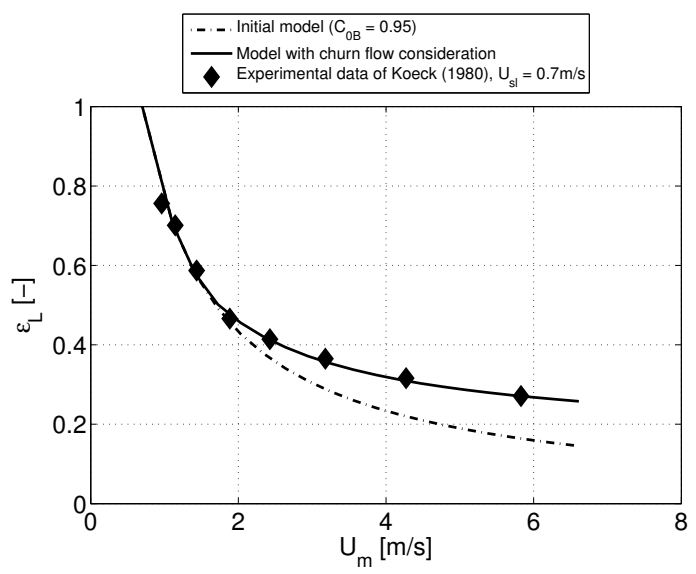

Fig. 14. Mean liquid fraction as a function of the mixture velocity, experimental data of Koeck (1980) for $U_{s l}=0.7 \mathrm{~m} \cdot \mathrm{s}^{-1}$. Comparison with the predictions obtained by taking into account the homogeneous to heterogeneous flow transition. The predictions are clearly improved in comparison with the initial model (see also figure 10). 


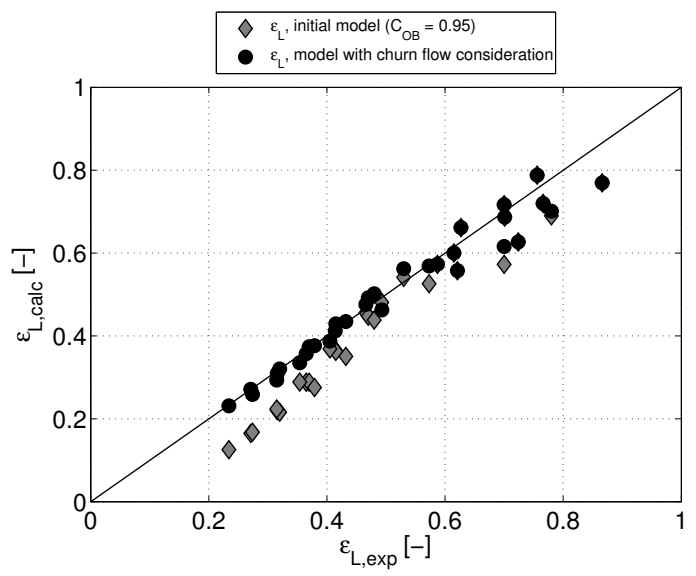

Fig. 15. Predicted mean liquid fraction as a function of the measured liquid fraction. All the experimental data of Koeck (1980) and of Fréchou (1986) for air-water flow are used. The precision of the homogeneous bubbly flow model and of the improved model taking into account the transition to churn flow are compared.

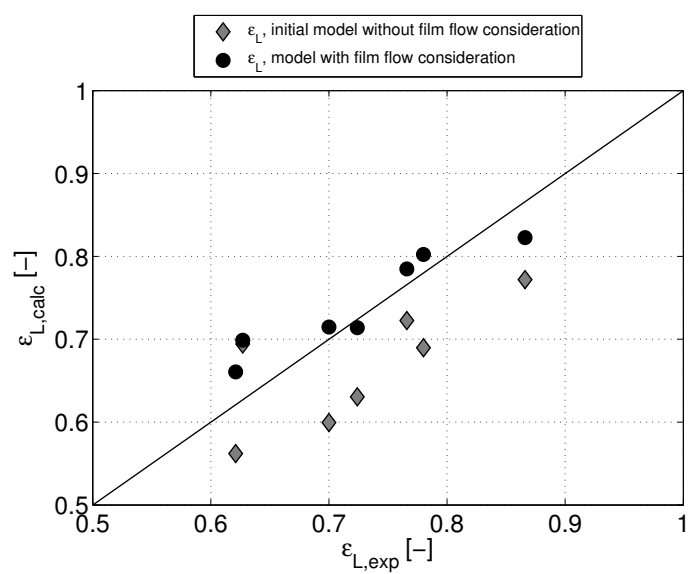

Fig. 16. Predicted mean liquid fraction as a function of the measured liquid fraction. The experimental data of Fréchou (1986) for oil are used $\left(\nu_{L}=30 \mathrm{mPa} . \mathrm{s}\right)$. The predictions with the initial model and with the improved film flow dependent model are compared. 